\title{
Fatigue-Life Computational Analysis for the Self-Expanding Endovascular Nitinol Stents
}

\author{
M. Grujicic, B. Pandurangan, A. Arakere, and J.S. Snipes
}

\author{
(Submitted December 18, 2011)
}

\begin{abstract}
Self-expanding endovascular stents made of Nitinol (a Ni-Ti intermetallic compound possessing superelastic and shape-memory properties) are being widely used to treat a common circulatory problem in which narrowed arteries, primarily due to fatty deposits, hamper blood flow to the extremities (the problem commonly referred to as "peripheral artery disease"). The stents of this type unfortunately occasionally fail structurally (and, in turn, functionally) rendering the stenting procedure ineffective. The failure is most often attributed to the fatigue-induced damage since over its expected ten-year life span, the stent will normally experience 370-400 million pulsating-blood flow-induced loading cycles. Redesign/redevelopment of the stents using the conventional make-and-test approaches is quite expensive and time consuming and therefore is being increasingly complemented by computational engineering methods and tools. In the present study, advanced structural and fluid-structure interaction finite element computational methods are combined with the advanced fatigue-based durability analysis techniques to further enhance the use of the computational engineering analysis tools in the development of vascular stents with improved high-cycle fatigue life.
\end{abstract}

Keywords fatigue-life prediction, Nitinol, stents

\section{Introduction}

The main objective of the present study is to investigate computationally fatigue-controlled service life of the selfexpanding Nitinol vascular stents. Hence, the key aspects of the present study are (a) Nitinol; (b) self-expanding vascular stents; and (c) fatigue-controlled service life of bio-medically implanted devices. These aspects will be briefly overviewed in the remainder of this section.

\subsection{Nitinol}

Nitinol is a binary metallic alloy (or more precisely, a binary inter-metallic) containing nearly identical atomic fractions of Nickel and Titanium. The alloy was discovered in 1961 during the course of a Naval Ordinance Laboratory (NOL) research project (Ref 1). The name Nitinol is derived from the chemical symbols of its two constituents (Ni and $\mathrm{Ti}$ ) and the abbreviation (NOL) of the research institution where the alloy was initially developed. The following Nitinol characteristics are often being cited as the main advantages being offered by this alloy: (a) high-level of biocompatibility which is critical in biomedical applications (Ref 2-5); (b) large extent of magnetic resonance imaging (MRI) opacity which enables imaging, using x-ray and MRI techniques (Ref 6,7). This characteristic makes Nitinol

M. Grujicic, B. Pandurangan, A. Arakere, and J.S. Snipes, Department of Mechanical Engineering, Clemson University, 241 Engineering Innovation Building, Clemson, SC 29634-0921. Contact e-mail: gmica@clemson.edu. ideally suited for use in biomedical implantable devices; and (c) unique thermo-mechanical response associated with the presence of the so-called shape-memory and superelasticity phenomena (defined below).

Since its introduction into the commercial market in the 1970s, Nitinol has found its use in a variety of applications, such as earthquake dampers, eyeglasses, orthodontic wires, pipeline couplings, bra underwires, phone antennas, microactuators, etc. (Ref 6, 8-10). In recent years, however, biomedical devices have become the main area of application/use of Nitinol (Ref 6, 9, 11).

The unique mechanical characteristics of Nitinol mentioned above are the result of a first-order diffusionless displacive phase transformation which converts a simple cubic-ordered $B 2$ parent-phase crystal structure into a monoclinic-ordered $B 19^{\prime}$ martensite-phase crystal structure (Ref 12, 13). This transformation is of an athermal character and occurs at a very high rate, once the thermodynamic conditions for the onset of transformation are achieved. The martensitic transformation in question can be induced by purely thermal means (via cooling below a temperature, denoted as the martensite-start, $M_{\mathrm{s}}$, temperature), by the application of stress or by a combination of the two. In addition to $M_{\mathrm{s}}$, there are two more characteristic temperatures $M_{\mathrm{f}}$ and $M_{\mathrm{d}}$ associated with the parent-phase to martensite transformation. $M_{\mathrm{f}}$ represents the highest temperature at which thermally induced martensitic transformation is complete. On the other hand, $M_{\mathrm{d}}$ represents the lowest temperature at which the parent phase would undergo plastic deformation before experiencing a stress-induced martensitic transformation. During heating, thermally induced martensitic transformation can be driven in the opposite direction and the two characteristic temperatures associated with the martensite to parent-phase transformation are denoted as $A_{\mathrm{s}}$ and $A_{\mathrm{f}}$, where the symbol $A$ stands for austenite and is often used loosely to denote the parent-phase in question. Based on these considerations, 
it is clear that a stress-induced martensitic transformation can take place only in the $A_{\mathrm{f}}-M_{\mathrm{d}}$ temperature range.

Depending on the exact alloy chemistry, impurity level and material thermo-mechanical treatment, a third (rhombohedral) $R$-phase (Ref 14-17), in addition to the parent phase and martensite, can form during cooling/loading. This transient phase compromises the beneficial characteristics of the forward and reverse martensitic transformation and is normally undesirable in Nitinol alloys used in vascular stent applications, in particular, and in implantable biomedical devices, in general (Ref 18, 19).

While martensitic transformations are of a displacive character (i.e., dominated by the shear component of the strain accompanying the transformation) they are generally also accompanied by changes in the material volume. In majority of the martensitic transformations studied in the open literature, the transformation-induced volume change is positive (i.e., the material undergoes expansion during transformation). This fact has been exploited in some materials (e.g., in the so-called TRIP TRansformation-Induced Plasticity, steels) to reduce the extent of stress-triaxiality in the crack tip region, and, thus, improve materials' toughness/tensile-strength (Ref 20). In Nitinol, on the other hand, parent-phase to martensite transformation is associated with a small $(0.33-0.54 \%)$ negative volume change (Ref 21-23). The presence of a negative transformation volume change in Nitinol is frequently being cited as the main cause of its limited fracture toughness and fatigue resistance (Ref 21, 22).

Owing to the presence of the aforementioned thermally induced and stress-induced forward/reverse martensitic transformations in Nitinol, this alloy possesses unique mechanical behavior as typified by the phenomena of "shape memory" and "superelasticity."

Shape memory is the ability of Nitinol to remember its predeformation shape and restore it when the alloy, in its deformed state, is subjected to a sufficiently high temperature. A schematic is provided in Fig. 1 to explain the phenomenon of shape memory. From the $T_{1}>A_{\mathrm{f}}$ temperature region in which the parent-phase is thermodynamically stable, Nitinol is cooled to a temperature $T_{2}<M_{\mathrm{f}}$ causing a complete transformation of the parent phase into martensite. Then, while still at $T_{2}$, the material is deformed. The dominant deformation mechanism in this case is the motion of the twin boundaries within martensite which converts less favorably oriented martensite variants into the more favorable oriented ones. Upon unloading, this portion of the deformation remains in the material while only the elastic strains are recovered. When subjected to temperatures in excess of $A_{\mathrm{f}}$, a complete martensite to parent phase transformation takes place, while the material restores its pre-deformation shape. The ability of Nitinol to remember its pre-deformation shape is utilized in many bio-medical implantable devices (such as the Simon vena-cava filter). This implantable device is first cooled to below $M_{\mathrm{f}}$, collapsed, and placed into a pre-cooled catheter, inserted into the heart, and allowed to acquire its predetermined shape upon reaching the human body temperature (which exceeds $A_{\mathrm{f}}$ ) (Ref 24).

Superelasticity is the ability of Nitinol to undergo large recoverable/elastic strains (ca. 11\%) because of the operation of the forward and reverse stress-induced martensitic transformations in the $A_{\mathrm{f}}-M_{\mathrm{d}}$ temperature range. A schematic of the uniaxial stress-strain loading/unloading curve showing the effect of stress-induced martensitic transformation on the superelastic behavior of Nitinol is depicted in Fig. 2. Initially,

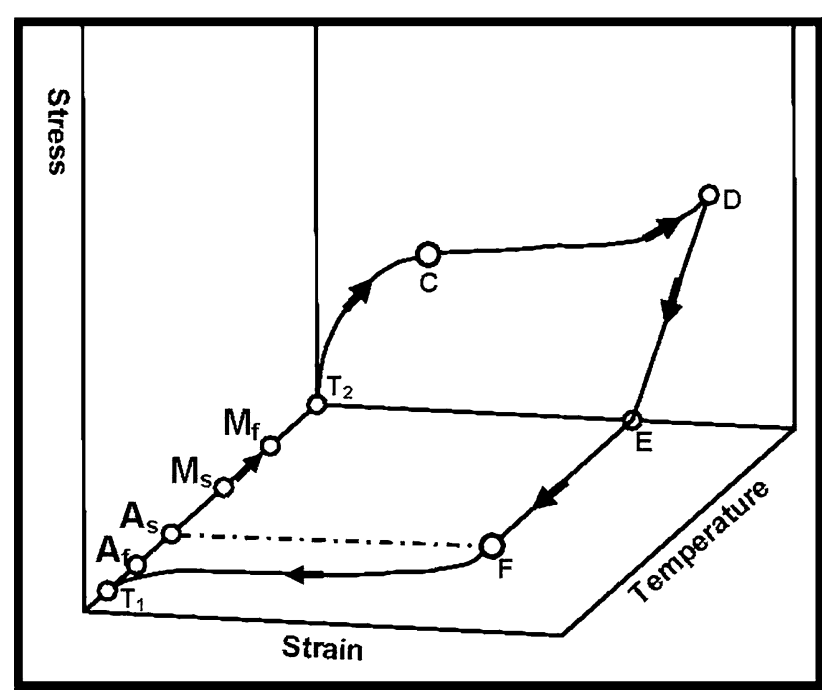

Fig. 1 A schematic of the shape-memory effect in Nitinol. At a temperature $T_{1}$ (e.g., the body temperature) which exceeds the austenite finish temperature $A_{\mathrm{f}}$, the alloy is stress free and fully austenitic. Upon cooling to temperature $T_{2}$ which is lower than the martensite finish temperature $M_{\mathrm{f}}$, the alloy remains stress free but transforms completely into martensite. During loading at $T_{2}$ along the $T_{2}$-C-D branch, martensite deforms by the motion of its straininvariant twin boundaries. Complete unloading along the D-E branch leaves a residual strain at temperature $T_{2}$. The residual strain survives heating up to the austenite start temperature $(\mathrm{F})$, and then it is completely eliminated during additional heating to $T_{1}$

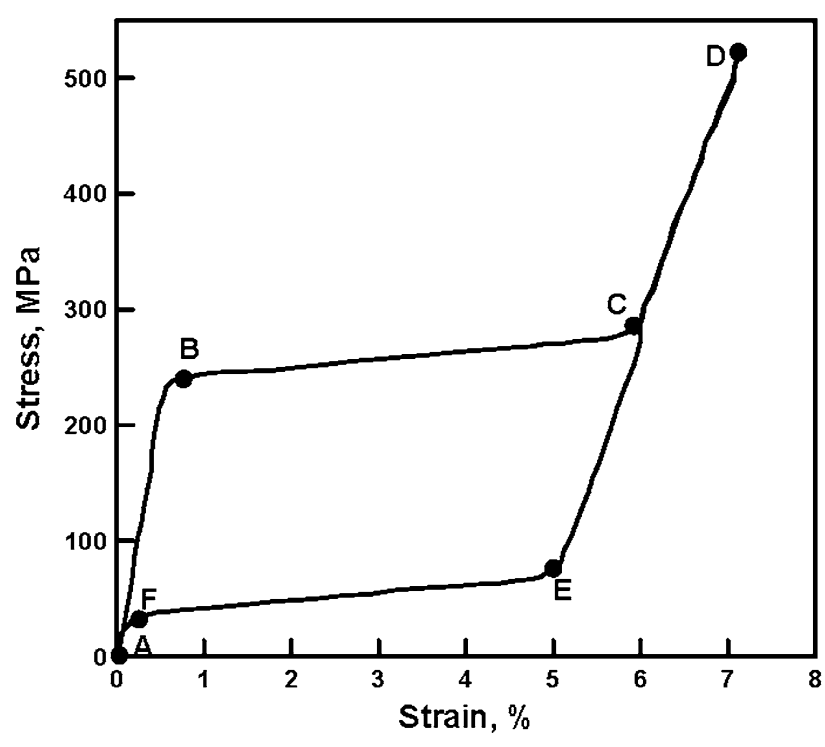

Fig. 2 Typical superelastic behavior of Nitinol observed at temperatures exceeding the austenite finish temperature but lower than martensite deformation temperature. The stress loading history is typified by the following points: A-the initial stress-free fully austenitic state; A-B austenite elastic loading; B-C forward martensitic transformation; C-D martensite elastic loading; D-E martensite elastic unloading; E-F reverse martensitic transformation; and F-A austenite elastic unloading to zero residual strain

the parent-phase undergoes purely elastic deformation. At a temperature-dependent stress level, however, martensite formation is initiated and continues to take place under a fairly 
constant stress level. Upon completion of the forward transformation, now fully martensitic material continues to deform elastically. During unloading of the material from the fully transformed state, elastic unloading of martensite first takes place. Then, the reverse martensite-to-parent-phase transformation is initiated and driven to completion (at a fairly constant stress level). Since the reverse transformation stress plateau is lower than the one associated with the forward transformation, a stress-strain hysteresis is formed, and finally, elastic unloading of the parent phase takes place leaving no residual strain in the material. As will be shown later, superelasticity of Nitinol is utilized in the so-called endovascular self-expandable stents.

\subsection{Self-Expanding Endovascular Stents}

The term "biomedical stents" typically refers to devices that are used for bracing or scaffolding diseased or traumatized human body vessels to (a) enable the flow of biological fluids by increasing the vessel flow cross section; and (b) prevent the collapse of the weakened vessel walls (Ref 25). While majority of the biomedical stents are used in cardiovascular applications, these devices are also employed in other parts of the human anatomy. For example, they are used to brace a collapsed trachea after a car crash or to reinforce the esophagus damaged by chronic gastric reflux (Ref 26).

As far as the endovascular stents are concerned, the ones used nowadays can be generally divided into two categories: (a) balloon-expandable permanently deformed stents typically made of stainless steel; and (b) self-expandable Nitinol stents. The latter endovascular stents are the subject of the present study for the following reasons: (a) they are generally found to exert a self-regulating low-magnitude outward force on to the vessel walls; (b) they are physiologically more compatible with blood vessels; and (c) they possess the ability to regain their shape after unintentional exposure to external forces as in the case of the superficial vessels (e.g., carotid artery). The adjective "endo" used here implies that these stents are introduced into the human body percutaneously via a catheter which is inserted into a large blood vessel (e.g., femoral artery or vein in the groin area) and delivered to the target location within the traumatized/diseased vessel.

In the case of self-expandable Nitinol vascular stents, advantage is taken of the superelastic behavior of Nitinol during both the deployment and in-vivo operation phases. To deploy the stent (which initially contains $100 \%$ of the Nitinol parent-phase), the stent is first crimped (i.e., its outer diameter is reduced by the application of radial forces) and placed in the delivery system at the tip of the catheter. To prevent premature deployment during delivery into the body, the stent is appropriately restrained. Once the catheter tip is inserted into the vessel and its tip guided to the target location, the stent is ejected from the delivery system and allowed to expand. The reverse martensite-to-parent phase transformation begins to take place as the stent expands and begins to conform to vessel walls.

To help us explain the stent stress history during its crimping, deployment and re-expansion/vessel-interaction phases, a simple radial force vs. stent diameter plot is shown in Fig. 3. The pre-crimping and the crimped conditions of the stent are represented in this figure by points $A$ and $B$, respectively. During deployment, the stent expands to a larger diameter represented by the point $C$ in Fig. 3. It is seen that this process is accompanied by the reverse martensite-parent phase

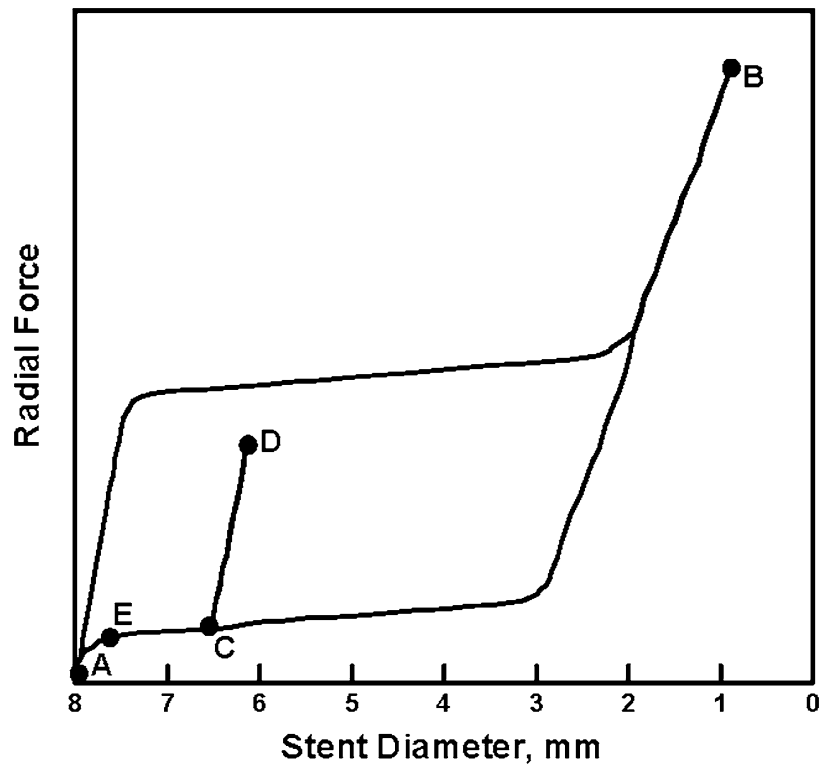

Fig. 3 The phenomenon of "biased-stiffness" occurring in selfexpanding Nitinol stents at room temperature. As-annealed, fully austenitic, stress-free over-sized stent (A) is crimped to a smaller diameter (B) and placed into the delivery system at the tip of the catheter. At the target location and upon deployment, the stent expands to a larger diameter (C). However, this expansion is not complete because of the resistance provided by the contacted artery wall, while the deployed stent continues to exert a mild outward force on the artery wall (C-E). Potential radial contraction of the stent due to external inward radial displacement is resisted by relatively large radial forces (C-D)

transformation. However, this transformation does not attain completion since the vessel walls prevent full expansion of the stent. Consequently, the stent continues to exert an outward radial force on the vessel walls which ensures that the vessel flow cross section remains at the desired level. It should be noted that the response of the stent at point $C$ is quite different to the outward and inward radial forces. The inward radial forces are confronted by a very stiff stent response ( $C-D$ load path) while the expansion of the stent in the radial direction is quite compliant $(C-E)$ load path. This phenomenon is commonly referred to as "biased stiffness." This behavior makes the self-expanding Nitinol stents particularly effective in ensuring that the vessel allows unimpeded flow of bodily fluids while maintaining its structural integrity when subjected to external forces. In addition, the force hysteresis displayed in Fig. 3 allows the stent to continuously exert a force of relatively low magnitude on the vessel wall despite potentially large deflections of the vessel walls and expansion of the stent.

\subsection{Fatigue-Controlled Service Life}

As discussed above, self-expanding endovascular Nitinol stents are effective in treating peripheral artery disease (a common circulatory problem in which narrowed arteries, primarily due to fatty deposits, hamper blood flow to the extremities). Unfortunately, stents occasionally fail while in service rendering the stenting ineffective. This finding is not that surprising considering that, in the course of ten-year expected service life of a stent, the stent would experience around 370-400 million cycles because of the pulsating blood flow through the artery. What is more concerning is the fact that 
a significant fraction of the deployed stents may fail within the first year of service. The more frequent failure occurrences should be rectifiable through modifications in the stent design. Direct experimental validation of the improved fatigue strength of the altered stent designs is, however, a time-demanding and relatively costly procedure. To shorten the stent development cycle and reduce the associated cost, computer engineering methods and tools are being increasingly utilized. There are several examples in the open literature demonstrating the benefits offered by the computer engineering methods and tools within the vascular stent (Ref 27-30).

Experimental investigations of the stent fatigue behavior identified two main contributing factors which control stent life: (a) radial cyclic expansion/contraction of the stented artery due to the pulsating blood flow; and (b) stent oversizing, i.e., the difference between the pre-crimping stent diameter and the artery inner diameter. Through proper selection of the testsample geometry, experimental cyclic-loading procedure, and data reduction (and in combination with the nonlinear finite element analysis), the appropriate strain amplitude vs. fatigue life and constant life strain amplitude vs. mean strain relations for Nitinol are established (Ref 30-32). These plots establish (a) a continuous decrease (at a progressively lower rate) of the maximum principal-strain amplitude with an increase in the fatigue cycle number; and (b) a weak (and potentially negative) dependence of the high-cycle fatigue portion of the strainamplitude vs. fatigue cycle number curve on the mean-strain magnitude (which is contrary to observations made in the majority of engineering materials) (Ref 32).

The main objective of the present study is to further the application of the computer-aided engineering methods and tools in the development of self-expanding endovascular Nitinol stents with enhanced fatigue-controlled service life. This article structure is organized as follows: Brief descriptions of the Lagrangian and the Combined Eulerian-Lagrangian (CEL) computational analyses used in the present study are provided in sections 2.1 and 2.2, respectively. The problem definition involving a stented artery subjected to the pulsating blood flow and the outward radial force conditions is presented in section 2.3. The main results are presented and discussed in section 3 . The key conclusions resulting from the present study are summarized in section 4 .

\section{Problem Formulation and Computational Procedure}

As mentioned earlier, the problem analyzed in the present study deals with a computational engineering analysis of the manufacture, deployment, and in-vivo service-performance assessment of a prototypical self-expanding endovascular Nitinol stent. The service performance of the stent is analyzed using two distinct computational formulations: (a) a pure Lagrangian formulation within which the blood is not modeled explicitly, but its presence is accounted for by prescribing an oscillatory/pulsating pressure boundary conditions to the vessel/artery walls in a range in accordance with the typical diastolic $10.6 \mathrm{kPa} \quad(80 \mathrm{mmHg})$ and systolic $15.9 \mathrm{kPa}$ (120 $\mathrm{mmHg}$ ) pressures and the pulse rate ( 72 beats per minute) of a human; and (b) a CEL analysis within which the blood is considered explicitly, and its pulsation is modeled by prescribing the appropriate velocity and pressure oscillatory boundary conditions at the inlet/outlet of the artery segment being analyzed. For improved clarity, the two types of analyses are presented henceforth in separate sections, while care is taken to prevent redundant descriptions/formulations.

All the calculations carried out in this study were done using either ABAQUS/Standard (Ref 33) or ABAQUS/Explicit computational codes (Ref 34). ABAQUS/Standard was used to carry out a quasi-static Lagrangian analysis, while ABAQUS/Explicit was used to conduct the dynamic CEL analysis.

\subsection{Lagrangian Analysis}

Within a prototypical Lagrangian (finite element) analysis, the computational mesh is attached to, and moves and deforms with, the material. Each finite element can contain only one material and the material permanently resides within its host element. A Lagrangian analysis generally involves the specification of the following: (a) the geometrical/meshed models for the computational domain constituents (the stent and an artery segment, in the present case); (b) constitutive relations for all the constituent materials; (c) the equations defining the initial, boundary, loading, contact and kinematic-constraint conditions; and (d) a numerical algorithm used to solve simultaneously the governing (mass conservation, linear momentum, and energy balance) equations along with the material constitutive equations and the equations defining the initial, boundary, loading, contact, and kinematic-constraint conditions.

2.1.1 Meshed Models. The computational domain used here consists of two Lagrangian components, a vascular stent and an artery segment. The meshed model for the vascular stent, Fig. 4(a), consists of 9312 hexahedron first-order continuum elements and was downloaded from Ghent University (www.stentibitech.ugent.be/downloads/downloads). The key dimensions of the stent before its radial expansion are indicated in Fig. 4(a). The artery segment analyzed, Fig. 4(b), was meshed using 3200 fournode first-order shell elements. The key dimensions of the artery segment before stent deployment are depicted in Fig. 4(b). A mesh sensitivity analysis was carried out (the results not shown for brevity) to ensure that the results obtained are not significantly affected by a further refinement of the mesh.

2.1.2 Material Models. The thermo-mechanical behavior of Nitinol is represented using the material model built-in into the ABAQUS material library. The model is the implementation of the generalized plasticity-based formulation initially proposed by Auricchio and Taylor (Ref 35, 36). Within the model, the total strain increment is partitioned into its linear elastic- and stress-induced transformation components. The stress-induced transformation strain components are derived using the conventional plasticity methodology. In other words, the concepts of transformation-controlled yield criterion and a plastic potential-based flow-rule are used. Specifically, forward and reverse martensitic transformation, temperature-dependent surfaces are defined analogous to yield surfaces in conventional plasticity theory. However, unlike the conventional pressureinvariant yield surface, transformation surfaces are taken to depend on the attendant pressure (to account for the transformation volume change). Also, the model accounts for the differences in the linear elastic behavior's constitutive response of the parent-phase and martensite.

The artery was modeled as a linear elastic material. In accordance with Tittelbaugh et al. (Ref 37), the Young's modulus and the Poisson's ratio for a prototypical diseased artery are set to $E=3.684 \mathrm{MPa}$ and $v=0.27$, respectively. 

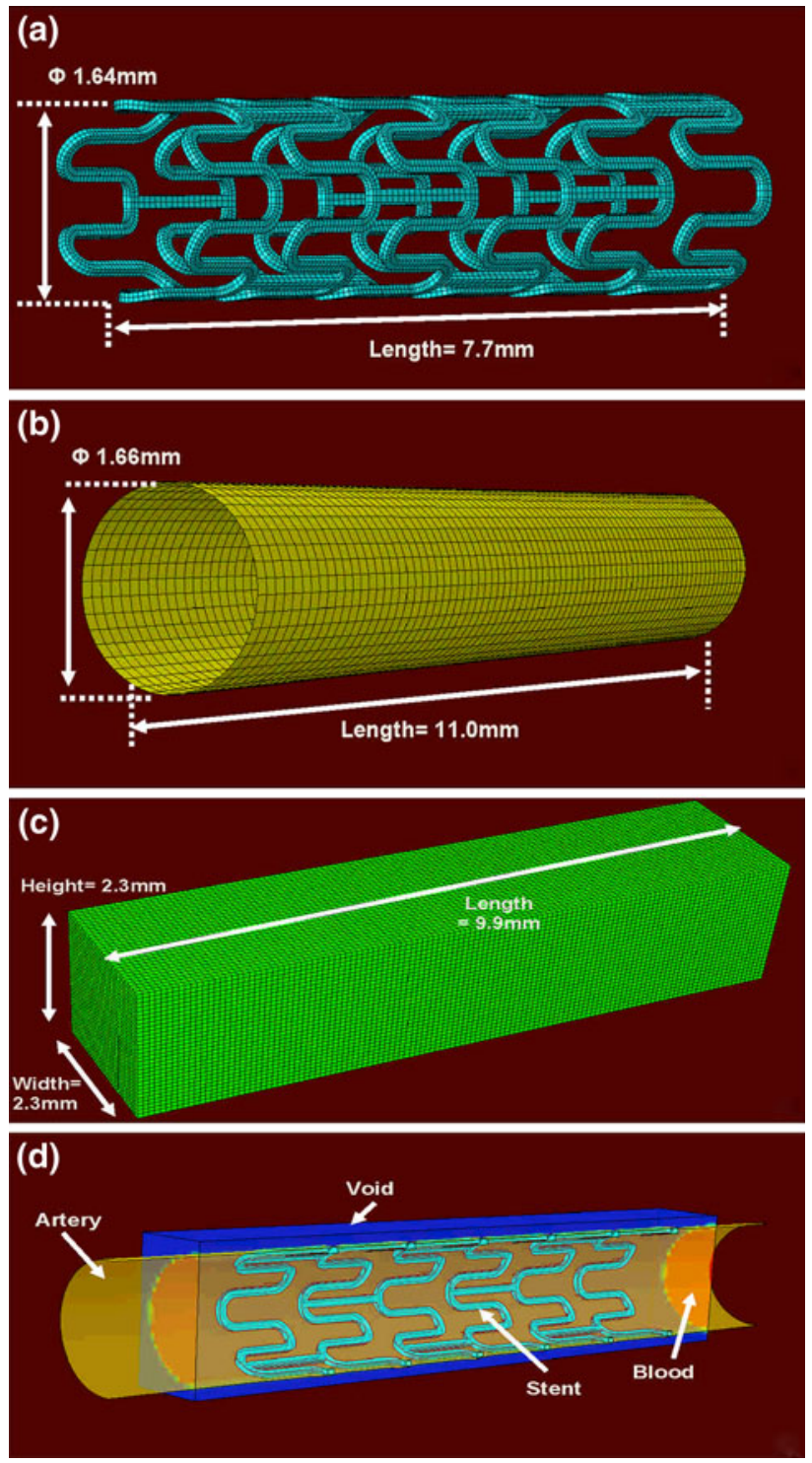

Fig. 4 Meshed models for: (a) the stent; (b) the artery segment; and (c) the associated Eulerian domain. The assembled combined Eulerian-Lagrangian geometrical models are shown in (d)

2.1.3 Initial Conditions. At the beginning of the simulation, all stresses within the stent and the artery are set to zero.

2.1.4 Boundary Conditions. Constant boundary conditions corresponding to the zero-displacement of the artery endnodes along the artery axis (the $z$-direction) are used throughout all the steps of the present analysis. As far as the stent boundary conditions are concerned, they were modified through the different steps of the present analysis. Specifically, (a) within the first step, stent radial expansion is modeled. This was accomplished by creating a cylindrical rigid surface which fits into the stent interior and by expanding this surface in the radial direction while ensuring its contact with the interior surface of the stent; (b) within the stent annealing stage, all the stent nodes are kept fixed while the expansion-induced stresses are removed; (c) stent insertion into the catheter is modeled by creating a cylindrical rigid surface which encloses the stent exterior and by contracting this surface in the radial direction, while ensuring its contact with the exterior surface of the annealed stent; (d) stent deployment into the artery is modeled by removing the rigid-surface/stent contacts while activating the stent/artery contacts. Also in this step, to prevent rigid body motion of the stent, translational degrees of freedom of two of its end nodes are constrained; and (e) during the in-vivo service-performance analysis step, the same boundary conditions were used as in step (d).

2.1.5 Loading Conditions. Loading was applied only during the in-vivo service-performance analysis step. It was assumed that the initial condition of the artery corresponds to a mean pressure of $13.3 \mathrm{kPa}(100 \mathrm{mmHg})$; hence, the loading associated with a pulsating blood is modeled by prescribing a sinusoidal pressure history to the artery walls with a $2.6-\mathrm{kPa}$ $(20 \mathrm{mmHg})$ amplitude and a $1.2-\mathrm{Hz}$ frequency. Since the dynamic effects at this frequency are negligibly small, the pressure loading is applied in a quasi-static manner.

2.1.6 Contact Interactions. The stent/artery contacts are analyzed using the so-called contact-pair method. Normal contact interactions are modeled using the "penalty contact" algorithm. Within this algorithm, (normal) penetration of the contacting surfaces is resisted by a set of linear springs which produce a contact pressure that is proportional to the depth of penetration. Typically, maximum default values, which still ensure computational stability, are assigned to the (penalty) spring constants. Force equilibrium in a direction collinear with the contact-interface normal then causes the penetration to acquire an equilibrium (contact-pressure dependent) value. Within this approach, no contact pressures are developed unless (and until) the nodes on the "slave surface" contact/penetrate the "master surface." On the other hand, the magnitude of the contact pressure that can be developed is unlimited. As far as the tangential contact interactions (responsible for transmission of the shear stresses across the contact interface) are concerned, they are modeled using a modified coulomb-friction law. Within this law, the maximum value of the shear stresses that can be transmitted (before the contacting surfaces begin to slide) is defined by a product of the contact pressure and a static (before sliding) and a kinetic (during sliding) friction coefficient. In addition, to account for the potential occurrence of a sticking condition (sliding occurs by shear fracture of the softer material rather than by a relative motion at the contact interface), a maximum value of shear stress (equal to the shear strength of the softer material) that can be transmitted at any level of the contact pressure, is also specified.

2.1.7 Numerical Algorithm. As mentioned earlier, the Lagrangian analysis is cast as a quasi-static problem. In addition, no thermal effects are expected within this analysis. Hence, the governing conservation equations are reduced in the present case to a set of force-equilibrium equations which are readily solved using an implicit finite-element method [like the one implemented in ABAQUS/Standard (Ref 33)].

\subsection{Combined Eulerian-Lagrangian Analysis}

Within a prototypical CEL (finite element) analysis, the computational domain consists of both the Lagrangian components and an Eulerian domain. In contrast to the Lagrangian components, the mesh in the Eulerian domain is fixed in space (i.e., it does not move or deform). The mesh elements can contain multiple materials which could flow in and out of the elements. A CEL analysis generally involves the specification of the following: (a) the geometrical/meshed models for the Lagrangian constituents (the stent and an artery segment, in the present case) and the Eulerian domain; (b) constitutive relations 
for all the constituent materials; (c) the equations defining the initial, boundary, loading, contact and kinematic-constraint conditions; and (d) a numerical algorithm used to solve simultaneously the governing (mass conservation, linear momentum, and energy balance) equations along with the material constitutive equations and the equations defining the initial, boundary, loading, contact, and kinematic-constraint conditions.

Since the same Lagrangian components are used in the CEL and the Lagrangian analyses, only the additional aspects of the CEL analysis are presented below.

2.2.1 Meshed Models. A 2.3 by 2.3 by $9.9 \mathrm{~mm}$ rectangular parallelepiped Eulerian domain containing 34800 hexahedron first-order Eulerian elements is used in this portion of the study, Fig. 4(c). The size of the domain was selected so it completely encompasses the deployed stent and the affected portion of the artery. The Eulerian element size was chosen as a compromise between the computational cost and the ability to accurately capture blood/stent and blood/artery contact interactions. The portion of the Eulerian domain encapsulated by the artery and the side faces of the Eulerian domain are initially filled with blood. The remainder of the Eulerian domain is assumed to be devoid of any material. It should be further noted that the portion of the Eulerian domain occupied by the stent is also devoid of any Eulerian material. To help us understand the spatial relationship between the stent, artery, and the blood, Fig. 4(d) is provided in which the void material, blood, and the artery are made transparent. It should be noted that, in Fig. 4(d), for improved clarity, only a half (obtained after a axial-longitudinal cut) of the CEL computational domain is shown.

2.2.2 Material Models. To account for the nonlinear volumetric response of blood, separate constitutive equations are used to represent the hydrodynamic (pressure) and the deviatoric (shear) components of the stress field. The hydrodynamic portion of the material model is represented using the linear shock speed (Us) - particle velocity (Up) Equation of State (EOS) which relates pressure to the mass-density and the internal-energy density. The EOS parameters used were taken from Ref 38 The deviatoric part of the stress was represented using a Newtonian viscosity-based model in which the (constant) viscosity parameter is set to a value of $0.003 \mathrm{~Pa} / \mathrm{s}$ (three times the viscosity of water).

2.2.3 Initial Conditions. It should be noted that the CEL analysis is used only to model the in-vivo service performance of the stent. Hence, the conditions pertaining to the displaced coordinates of the artery and the stent, after stent deployment, and the associated stresses in the two Lagrangian components are used as the initial conditions within the CEL analysis. In addition, zero velocity initial conditions are prescribed to all the Lagrangian nodes and the Eulerian material points. In other words, before blood pulsation via the imposed boundary conditions is introduced, all the components of the CEL analysis are assumed to be quiescent.

2.2.4 Boundary Conditions. The Lagrangian component boundary conditions in the in-vivo service-performance analysis step discussed earlier within the Lagrangian analysis are also used in this portion of the study. As far as the Eulerian domain boundary conditions are concerned, non default boundary conditions are prescribed only onto the circular portions of this domain outlined by the intersection of the artery with the two Eulerian domain external faces orthogonal to the artery axis.
On one of these faces, time-dependent inlet velocity boundary conditions are prescribed, Fig. 5(a). These boundary conditions were taken from Ref 39 in which (a) Doppler Ultrasonography was used to determine the mid-femoral artery flow-rate waveform which is characterized by (i) a rapid rise time; (ii) a peak value which is approximately eight times the mean; (iii) a rapid decline to a peak reverse flow rate magnitude of which is approximately three times the mean; (iv) a longer secondary rise time to a substantially lower forward peak value; and (v) finally, a gradual decline of the flow rate to zero; (b) after digitizing the signal, the data are fitted to a five-term sin/ cosine Fourier series. The resulting Fourier series coefficients can be found from Ref 40 . It should be noted that the resulting mean and peak Reynolds numbers were 125 and 980, respectively, while the Womersley number is 4.7 . This finding suggests that the blood flow is in the laminar regime and that the contribution of the pulsating/transient effects to the flow field is minimal.
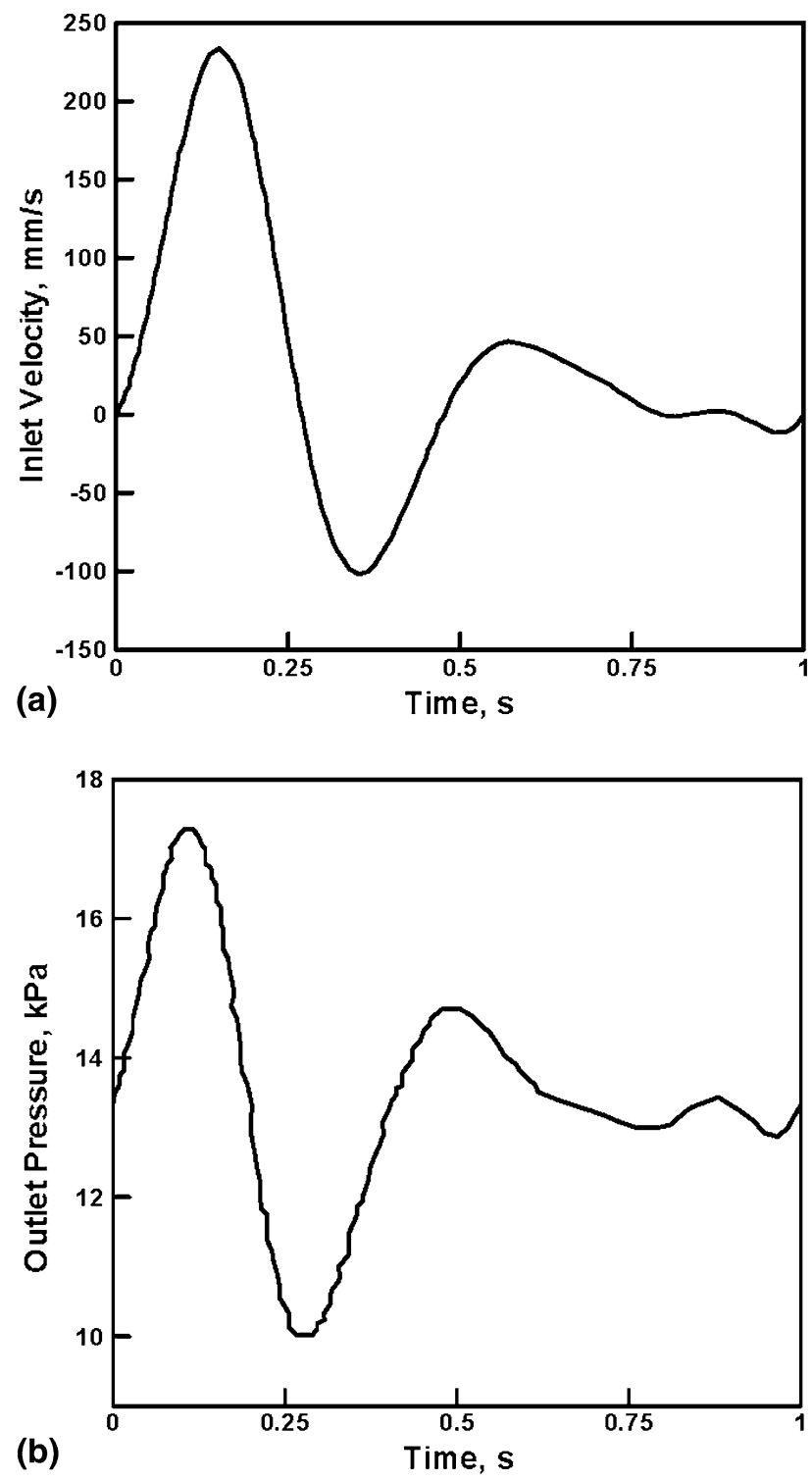

Fig. 5 Time-dependent: (a) inlet velocity; and (b) outlet pressure boundary conditions applied to the end faces of the artery-enclosed Eulerian section containing the blood 
On the other circular section of the Eulerian domain, timedependent outlet pressure boundary conditions were prescribed, Fig. 5(b). These were taken from Ref 39 in which: (a) computational fluid dynamics was used to analyze blood flow through a long vessel by prescribing the aforementioned timedependent inlet velocity and zero outlet pressure to the ends of a long vessel; (b) a cross section of the vessel is then identified at which the pressure amplitude is of the desired magnitude (ca. $2.6 \mathrm{kPa} / 20 \mathrm{mmHg}$ ); and (c) at this location, pressure is averaged over the entire cross section at each time step and the resulting pressure vs. time relation used in the present analysis.

2.2.5 Loading Conditions. No explicit loading conditions are used in the CEL analysis. However, loading associated with the pulsating blood is indirectly modeled through the blood/artery and blood/stent contact interactions.

2.2.6 Contact Interactions. Since the Eulerian domain and the Lagrangian components (stent and artery) do not possess conformal meshes, the contact interfaces between the two could not be defined using mesh-based surfaces. Instead, Lagrangian/Eulerian contact interfaces are determined using the so-called immersed boundary method (Ref 35). This "interface reconstruction" algorithm tracks, during each computational time increment, the position of Eulerian material boundaries in contact with the Lagrangian component(s). This is accomplished by approximating the material boundaries within an element as simple planar facets. Consequently, accurate determination of the Eulerian boundaries requires the use of fine Eulerian meshes.

Eulerian-Lagrangian contact normal interactions are enforced using a penalty method, within which the extent of contact pressure is governed by the local surface penetrations (where the default penalty stiffness parameter is automatically maximized subject to stability limits). As far as the shear stresses are concerned they are transferred via a "slip/stick" algorithm, that is shear stresses lower than the frictional shear stress are transferred without interface sliding (otherwise interface sliding takes place). The frictional shear stress is defined by a modified Coulomb law within which there is an upper limit to this quantity.

2.2.7 Numerical Algorithm. The governing mass conservation, linear momentum and energy balance equations along with the material constitutive equations and the equations defining the initial, boundary, loading, contact and kinematicconstraint conditions are solved over the entire CEL computational domain using a conditionally stable second-order explicit numerical procedure [as implemented in ABAQUS/ Explicit (Ref 34)]. It should be noted that the numerical solution of the governing equations in the Eulerian portion of the CEL computational domain within each time increment involves two separate steps: (a) the Lagrangian step within which the sub-domain is temporarily treated as being of a Lagrangian-type (i.e., nodes and elements are attached to and move/deform with the material); and (b) the "remap" step within which the distorted mesh is mapped onto the original Eulerian mesh and the accompanying material transport is computed and used to update the Eulerian-material states and inter-material boundaries. As mentioned earlier, the explicit method used is conditionally stable and scales with the ratio of the element smallest dimension and the square root of the bulk modulus. Since small Eulerian elements had to be used for proper Euler-Lagrange contact identification and blood is nearly incompressible (i.e., possesses a large value of the bulk modulus), it is the Eulerian portion of the CEL analysis which controls the computational cost.

\subsection{Problem Definition}

The main problem addressed in the present study can be defined as the identification of the locations in a prototypical self-expanding endovascular Nitinol stent which are associated with the most severe fatigue-induced damage conditions (i.e., maximum strain amplitude combined with a maximum magnitude of the mean strain) and the prediction of the stent service life. In order to capture mechano-structural state of the stent in its deployed condition, steps associated with stent radial expansion, annealing, crimping, and deployment are analyzed in addition to the one associated with the in-vivo service under pulsating-blood flow loading conditions.

\section{Results and Discussion}

\subsection{Stent Expansion, Annealing, Crimping, and Deployment}

As mentioned earlier, in order to correctly capture the geometry of the deployed stent and the state of the material within it, the main steps associated with the stent manufacture and deployment are analyzed before studying the stent in-vivo performance. Figure 6(a)-(d) shows the stent configuration in the (a): laser as-machined condition (inner radius $=0.81 \mathrm{~mm}$, outer radius $=0.83 \mathrm{~mm}$ ); (b) expanded and subsequently annealed condition (inner radius $=3.0 \mathrm{~mm}$ ); (c) crimped condition (outer radius $=1.65 \mathrm{~mm}$ ); and (d) the deployed condition. It should be noted that in Fig. 6(c) and (d), the artery segment analyzed is shown in addition to the stent configuration. It is seen that due to the outward radial force exerted by the self-expanding endovascular stent, the artery in Fig. 6(d) is expanded radially in the region which is in direct contact with the stent. This is accompanied by an increase in artery local cross-sectional area which was the main objective of the stenting procedure.

\subsection{Comparison of the Lagrangian and the CEL Analyses}

The in-vivo performance of the self-expanding endovascular Nitinol stent is analyzed in the present study using two distinct computational approaches: (a) a Purely structural Lagrangian analysis within which the pulsating blood flow is not explicitly modeled, but rather its effect on the artery walls and, in turn, on the stent is accounted for; and (b) a Combined EulerianLagrangian fluid structure interaction analysis within which the blood and its pulsating flow are analyzed explicitly. The main reason for employing two distinct formulations is that the Lagrangian analysis is computationally much more efficient (computational times are lower by at least one order of magnitude) relative to the CEL analysis. Hence, it is important to establish to what extent the less accurate computationally more efficient Lagrangian analysis can be used as a substitute for the more accurate, computationally more expensive CEL analysis. Selected corresponding results obtained using the two analyses are presented and discussed in this section to answer this question.

As was discussed earlier, fatigue-controlled performance of the self-expanding vascular stents is mainly affected by the 

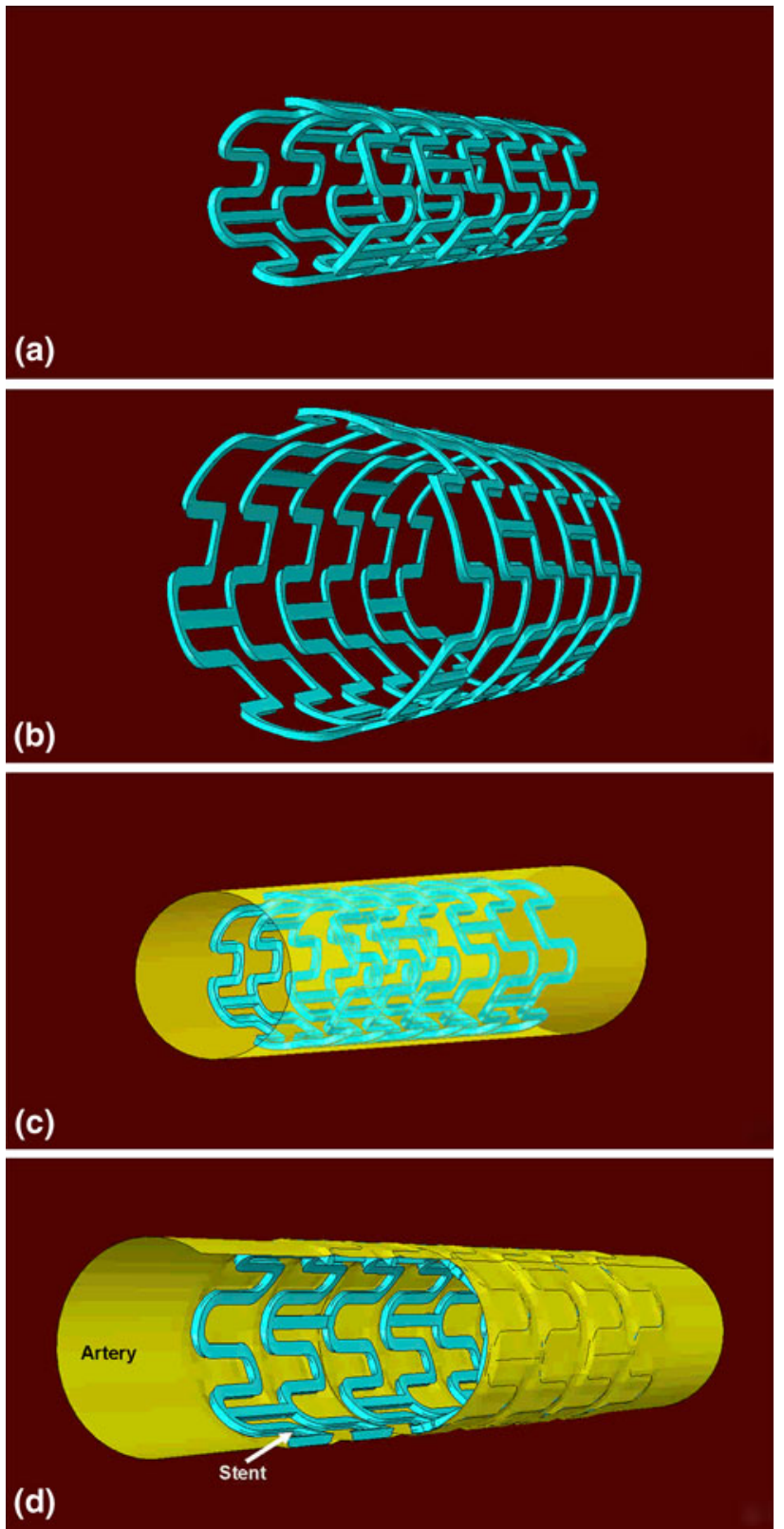

Fig. 6 Stent configurations in (a): laser as-machined condition; (b) expanded and subsequently annealed condition; (c) crimped condition; and (d) the deployed condition

mean value of the maximum principal strain and the local maximum principal-strain amplitude. Hence, in order to establish the extent of agreement between the two analyses, spatial distribution and temporal evolution of these two quantities throughout the stent are monitored.

A comparison of the maximum principal strain results obtained using the two analyses at the time within the loading cycle at which the pressure experiences its diastolic trough minimum value is given in Fig. 7(a) and (b). It should be noted that when the pressure experiences its minimum value, the artery acquires its smallest radius and, consequently, the strains experienced by the stent are the highest. A comparison of the results displayed in Fig. 7(a) and (b) suggests that, considering the fact that in the CEL analysis the pressure varies along the length of the artery, while in the Lagrangian case, it is constant,
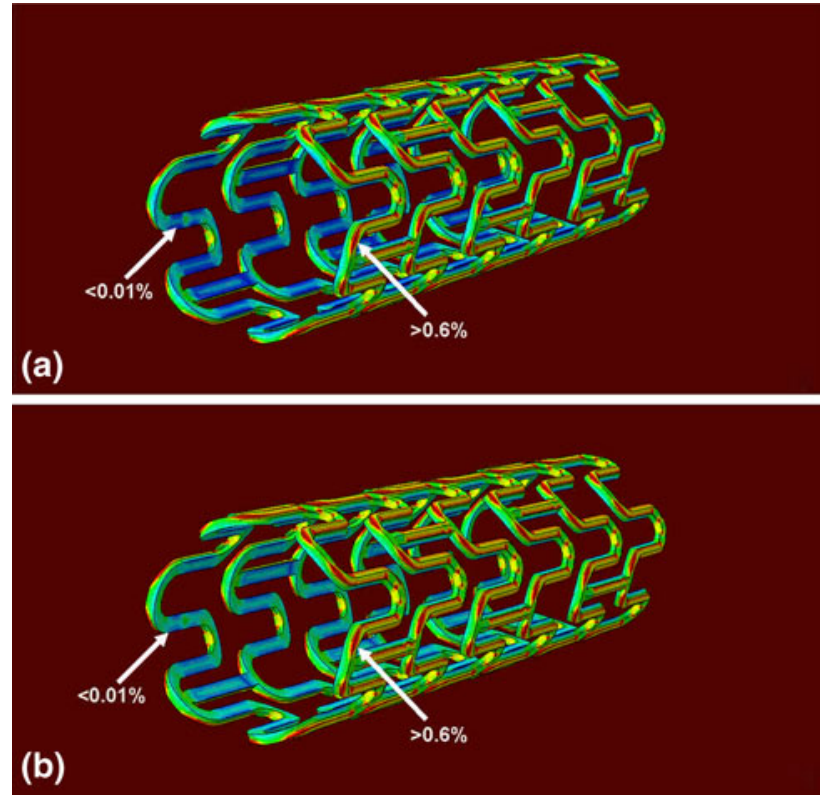

Fig. 7 Maximum principal strains within the stent at diastolic pressure obtained in (a) the Lagrangian; and (b) the CEL analyses

the two analyses yield comparable results relative to the maximum principal strain distribution within the stent at the diastolic pressure troughs. It should be also noted that the results displayed in Fig. 7(a) and (b) are obtained after ten loading cycles. The maximum principal strain distribution changes over the first few loading cycles but acquires a steady character by the beginning of the tenth cycle. The initial transient nature of the results obtained can be attributed to the combined effect of material nonlinearities and contact conditions.

A comparison of the maximum principal-strain amplitude results obtained using the two analyses are given in Fig. 8(a) and (b). These amplitudes are obtained using the corresponding maximum principal strain values at the systolic peaks and diastolic pressure troughs. The results displayed in Fig. 8(a) and (b) suggest that the two analyses yield comparable results relative to the maximum principal-strain amplitude distribution within the stent.

Thus, based on the results presented and discussed in this section, it appears that the Lagrangian analysis can be used as a reliable substitute for the CEL analysis, at least, while one deals with the calculations of the spatial distribution and temporal evolution of the maximum principal-strain mean and amplitude.

\subsection{Blood Circulating-Flow Analysis}

While the discussion presented in the previous section shows that with respect to the prediction of the spatial distribution and temporal evolution of the maximum principal mean strain and the maximum principal strain amplitude, the computationally more efficient Lagrangian analysis can be used with sufficient confidence in place of the computationally more expensive CEL analysis; nevertheless, the CEL analysis is intrinsically more accurate and can provide additional insight into the interaction of the circulating blood with the stent and the flexible artery. An example of the unique results obtained using the CEL analysis is presented in Fig. 9(a) and (d). The results presented in Fig. 9(a) and (b) correspond to the instant during the cardiac cycle when the pressure experiences the systolic peak, while the 

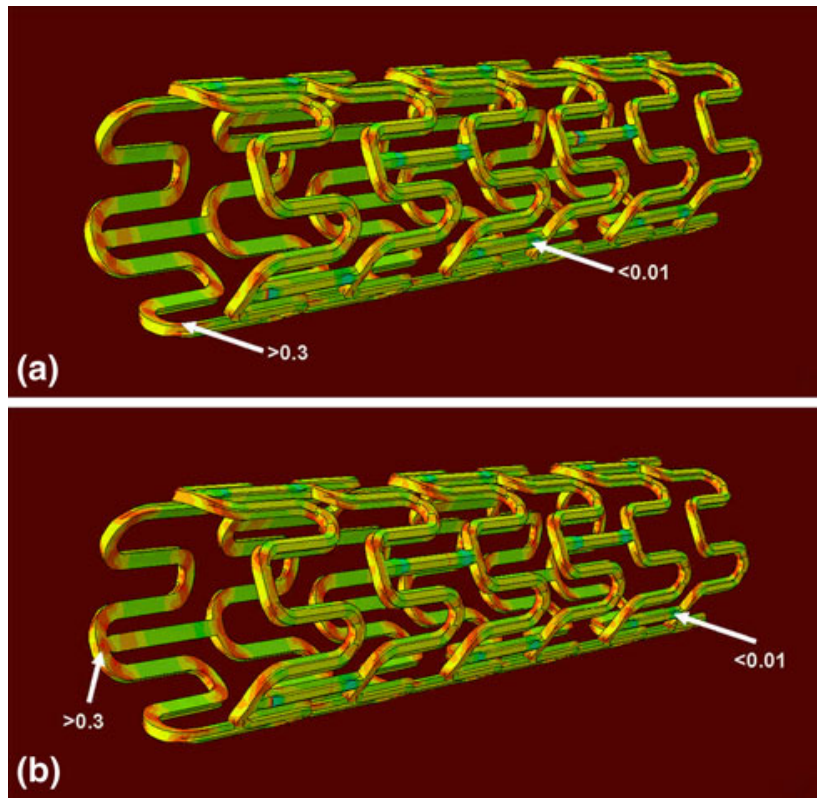

Fig. 8 Maximum principal-strain amplitude within the stent at the systolic peaks and diastolic pressure troughs obtained in (a) the Lagrangian; and (b) the CEL analyses

results displayed in Fig. 9(c) and (d) correspond to the instant during the cardiac cycle when the pressure experiences the diastolic trough. The results displayed in Fig. 9(a) and (c) pertain to the spatial distribution of the blood flow velocity over a midsection cut of the artery, while the results displayed in Fig. 9(b) and (d) pertain to the spatial distribution of the corresponding blood hydrostatic pressure over the same cut. The regions of the cut which are associated with the deployed stent as well as the inlet and outlet boundaries of the artery segment modeled are denoted in Fig. 9(a). An analysis of the results displayed in Fig. 9(a)-(d) shows:

(a) fairly complex velocity and pressure fields at both instances during the cardiac cycle;

(b) the expected parabolic distribution of the flow velocity over a portion of the artery section. In the portion of the artery section in which the velocity parabolic profile was not attained, the velocity was affected by the prescribed outlet pressure boundary conditions;

(c) There is an unexpected back flow of the blood in Fig. 9(a). This finding can be attributed to the fact that the inlet velocity and the outlet pressure boundary conditions used in the calculations came from different sources and, hence, they may not be fully consistent; and

(d) the conjecture made in part (c) can be supported by the results displayed in Fig. 9(b) which shows the upstream propagation of the pressure applied at the artery segment outlet.

\subsection{Stent Fatigue-Based Life Span Analysis}

Detailed experimental investigations carried out in Ref 32, 33 established that the high cycle fatigue behavior of Nitinol (of interest in the context of endovascular stents) is controlled by the local maximum principal-strain amplitude and the mean value of the maximum principal strain. These findings are
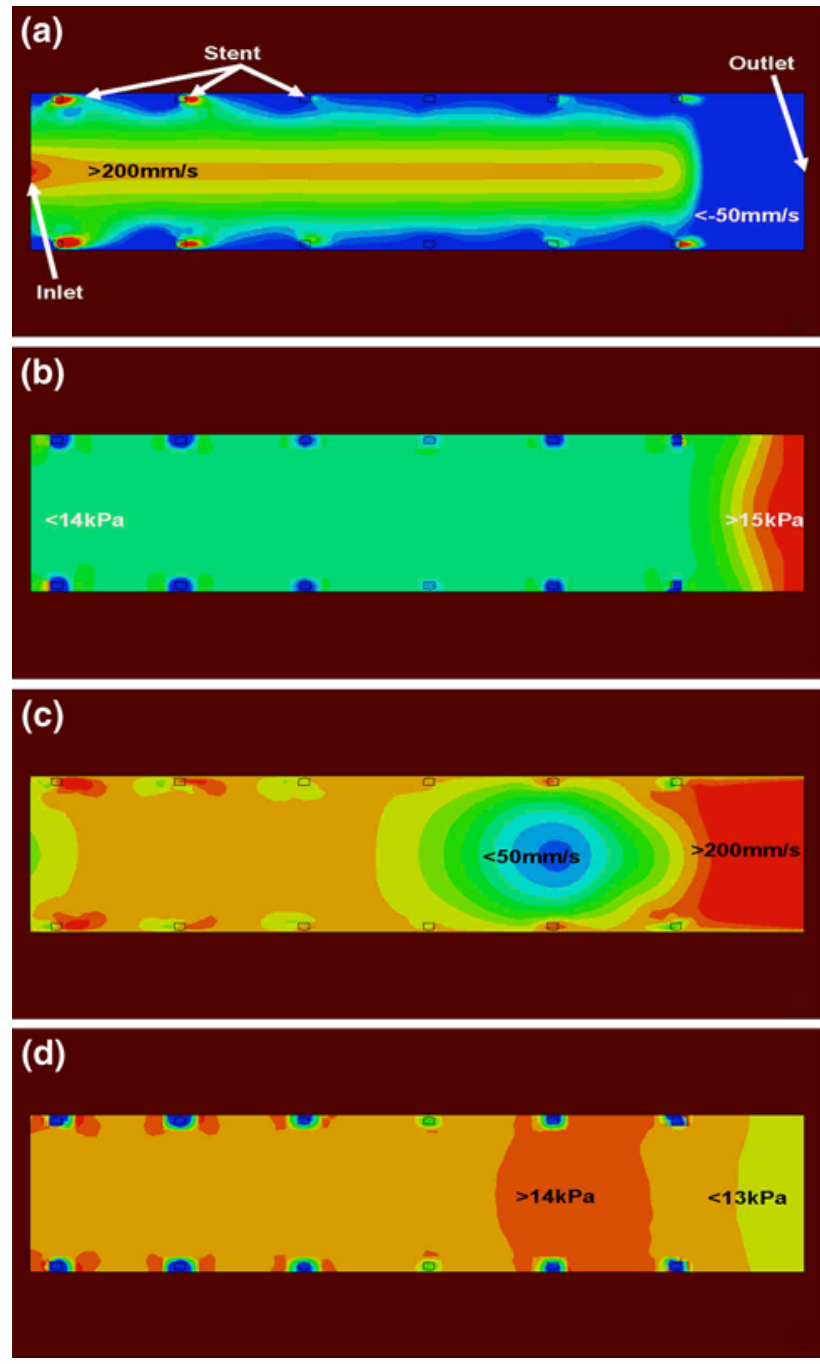

Fig. 9 CEL analysis-based results pertaining to the spatial distribution of (a) axial velocity; and (b) hydrostatic pressure of the blood at the systolic pressure peak moment within a cardiac cycle. (c, d) are the corresponding velocity and pressure results at the diastolic pressure trough, respectively

summarized in Fig. 10(a) and (b) in which (maximum principal) strain-amplitude vs. fatigue life, at a constant (zero) value of the (maximum principal) mean strain, and strain amplitude vs. the mean strain, at a constant $\left(10^{7}\right.$ cycles $)$ fatigue life, are shown, respectively. Examination of the results displayed in Fig. 10(a) shows that the strain amplitude decreases with the number of cycles till failure and apparently levels off to a value lower than $0.4 \%$, at a fatigue life in the range of several million cycles. As far as the results displayed in Fig. 10(b) are concerned, they indicate a fairly weak (potentially negative) effect of the magnitude of the maximum principal strain on the intensity of cyclic loading (as quantified by the maximum principal strain amplitude) at a constant level of the fatigue life.

The results presented in Fig. 10(a) and (b) pertain to the fatigue behavior of Nitinol as a structural material when subjected to constant strain-amplitude and constant mean strain loading conditions. However, the prediction of the fatiguecontrolled life span of a Nitinol stent from the knowledge of the Nitinol fatigue behavior is not a straight forward task. The main reason for this is the complex spatial distribution and temporal 

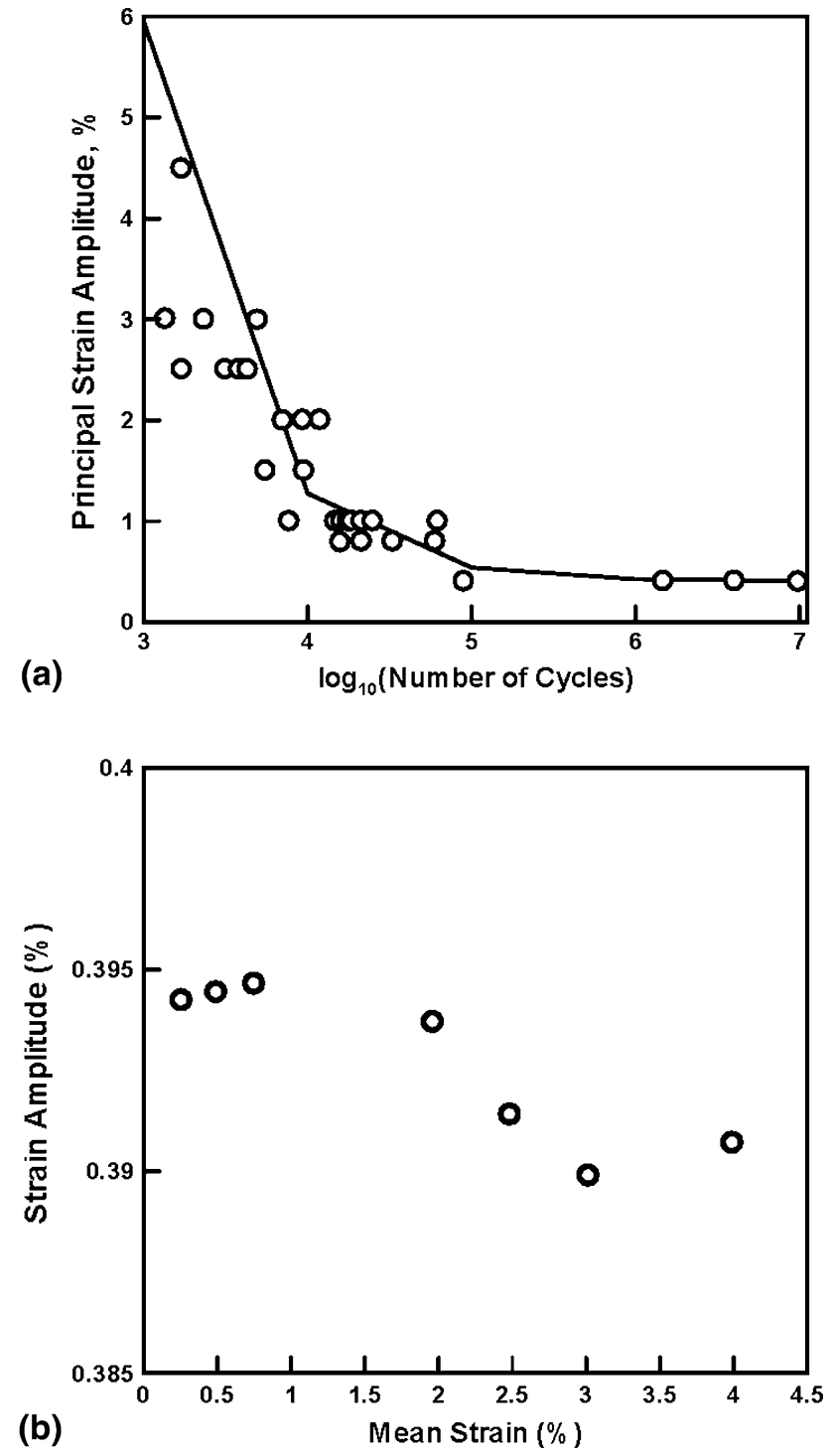

Fig. 10 (a) Maximum principal strain-amplitude vs. fatigue life at a constant (zero) value of the maximum principal mean strain; and (b) strain amplitude vs. the mean strain at a constant $\left(10^{7}\right.$ cycles $)$ fatigue life

evolution of the strains within the stent during a typical cardiac cycle. In other words, in-vivo loading observed at a typical location within the stent is of a non-constant strain amplitude and a non-constant mean strain character, while the available Nitinol fatigue data were obtained under constant strainamplitude cyclic loading conditions. To establish a correlation between the stent fatigue life and the Nitinol fatigue properties, a procedure is utilized in the present study which combines the so-called Rainflow loading cycle-counting analysis (Ref 41), a linearized Goodman diagram (e.g., Ref 42), to account for the effect of mean strain on the Nitinol fatigue life, and the Miner's linear-superposition principle/rule (Ref 43). Since this procedure was described in great detail in our recent study (Ref 44, 45 ), only a brief overview of its main components, i.e., the rainflow cycle-counting analysis, the Goodman diagram, and the Miner's rule, will be provided here.

3.4.1 Rainflow Cycle-Counting Analysis. When a timevarying load signal is recorded over a sampling period, and needs to be described in terms of a three-dimensional histogram (a)
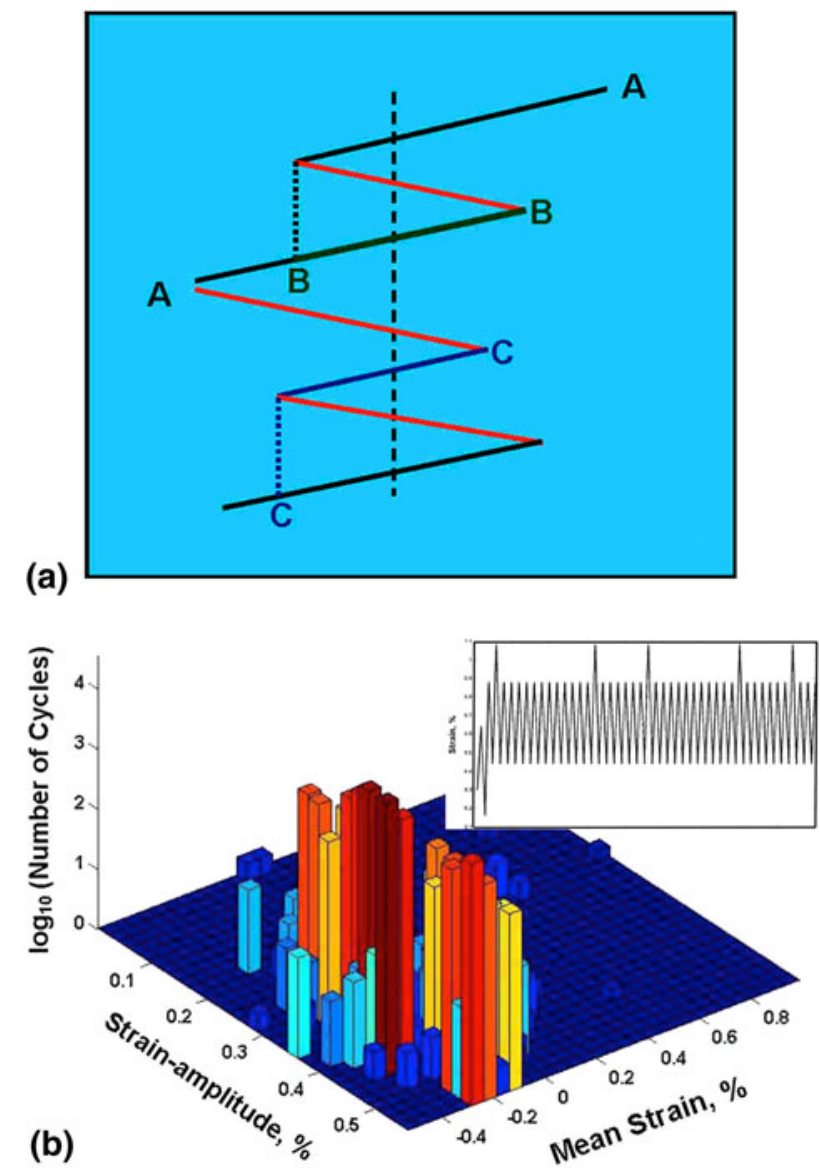

Fig. 11 (a) Application of the rainflow cycle-counting algorithm to a simple load signal after the peak/trough reconstruction. Please see text for explanation; and (b) the resulting three-dimensional histogram showing the number of cycles/half-cycles in each mean strainstrain amplitude bin

(each bin of which being characterized by a range of the signal amplitude and a range of the signal mean value), procedures, like the rainflow counting algorithm, are used. Within this procedure, the first step involves converting the original load signal into a sequence of load peaks and valleys. Then, the cycle-counting algorithm is invoked. To help us explain the rainflow cycle-counting algorithm, a simple load signal (after the peak/valley reconstruction) is depicted in Fig. 11(a), with the time axis running downward.

Within the rainflow cycle-counting algorithm, separate counting of load half-cycles is carried out for the ones starting from the peaks and the ones starting from the valleys. In Fig. 11(a), only the half-cycles originating from the peaks are analyzed. A half-cycle then starts from each peak and ends when one of the following three criteria is met:

(a) the end of the signal is reached (Case A in Fig. 11a);

(b) the half-cycle in question runs into a half-cycle which originated earlier and which is associated with a higher peak value (Case B in Fig. 11a); and

(c) the half-cycle in question runs into another half-cycle which originated at a later time and which is associated with a higher value of the peak (Case C in Fig. 11a).

Once all the half-cycles are identified, they are placed in bins, each bin being characterized by a range of the load 
amplitude and the load mean-value. A prototypical example of the resulting three-dimensional histogram showing the number of cycles/half-cycles present in the load signal associated with a given combination of the strain amplitude and the strain meanvalue is depicted in Fig. 11(b).

3.4.2 Goodman Diagram. Before presenting the basics of the Goodman diagram, it is important to recall that fatigue life of Nitinol is a function of both the strain amplitude and (to a lesser extent) the mean strain value. Often, the effect of mean strain is replaced by the effect of an $R$-ratio which is a ratio of the algebraically minimum and the algebraically maximum strain values (associated with the constant-amplitude cyclicloading tests). In other words, strain amplitude vs. fatigue life curves at a constant level of the mean strain are replaced with corresponding strain amplitude vs. fatigue life curves at a constant $R$-value. Using the definition of the mean strain amplitude and the $R$-value, it can be readily shown that fatigueloading tests carried out under constant $R$-ratio conditions, correspond to the tests in which the mean strain is scaled by the strain amplitude. To construct the Goodman diagram, constant$R$ lines are first generated within mean strain vs. strain amplitude diagram, Fig. 12(a). It is seen that the constant- $R$ lines are straight and all emanate from the origin. In Fig. 12(a), $R=0.1$ and $R=0.5$ data are associated with a positive/tensile mean stress/strain value, $R=-1$ corresponds to a zero meanvalue, while $R=10$ and $R=2$ pertain to a negative/compressive mean-value.

To construct the linearized Goodman diagram, the corresponding constant fatigue-life strain amplitude/mean strain points associated with neighboring constant $R$-ratio values are connected using straight lines. To complete the construction of the Goodman diagram, the constant fatigue-life lines are connected to the ultimate tensile strain and to the ultimate compressive strain points located on the zero-amplitude horizontal axis. A prototypical Goodman diagram is displayed in Fig. 12(a). The utility of this diagram is that for any combination of the strain amplitude and the mean strain, through the proper linear interpolation, one can determine the corresponding number of cycles to failure. Hence, using this diagram, one can construct a three-dimensional histogram analogous to the one shown in Fig. 11(b) except that the number of cycles in this case represents the total number of cycles to failure rather than the number of loading cycles. A prototypical three-dimensional histogram obtained using this approach is displayed in Fig. 12(b).

3.4.3 Miner's Rule. The cycle-counting procedure described earlier enables computation of the number of cycles/ half-cycles in the given load signal, which fall into bins of a threedimensional histogram, Fig. 11(b). The use of the Goodman diagram, on the other hand, enables the computation of the corresponding three-dimensional histogram, but for the number of cycles to failure (i.e., the fatigue life), Fig. 12(b). According to the Miner's rule, the ratio of the number of loading cycles and the corresponding maximum (before failure) number of cycles, for a given combination of the strain amplitude and strain mean-value, quantifies the fractional damage associated with this component of the cyclic loading. The total damage experienced by a material point after a given loading time is then obtained by summing the fractional damages associated with all components of the cyclicloading signal (i.e., with all combinations of strain amplitude and the strain mean-value).

The total fatigue life under the given non-constant amplitude cyclic loading is then obtained by dividing the loading duration
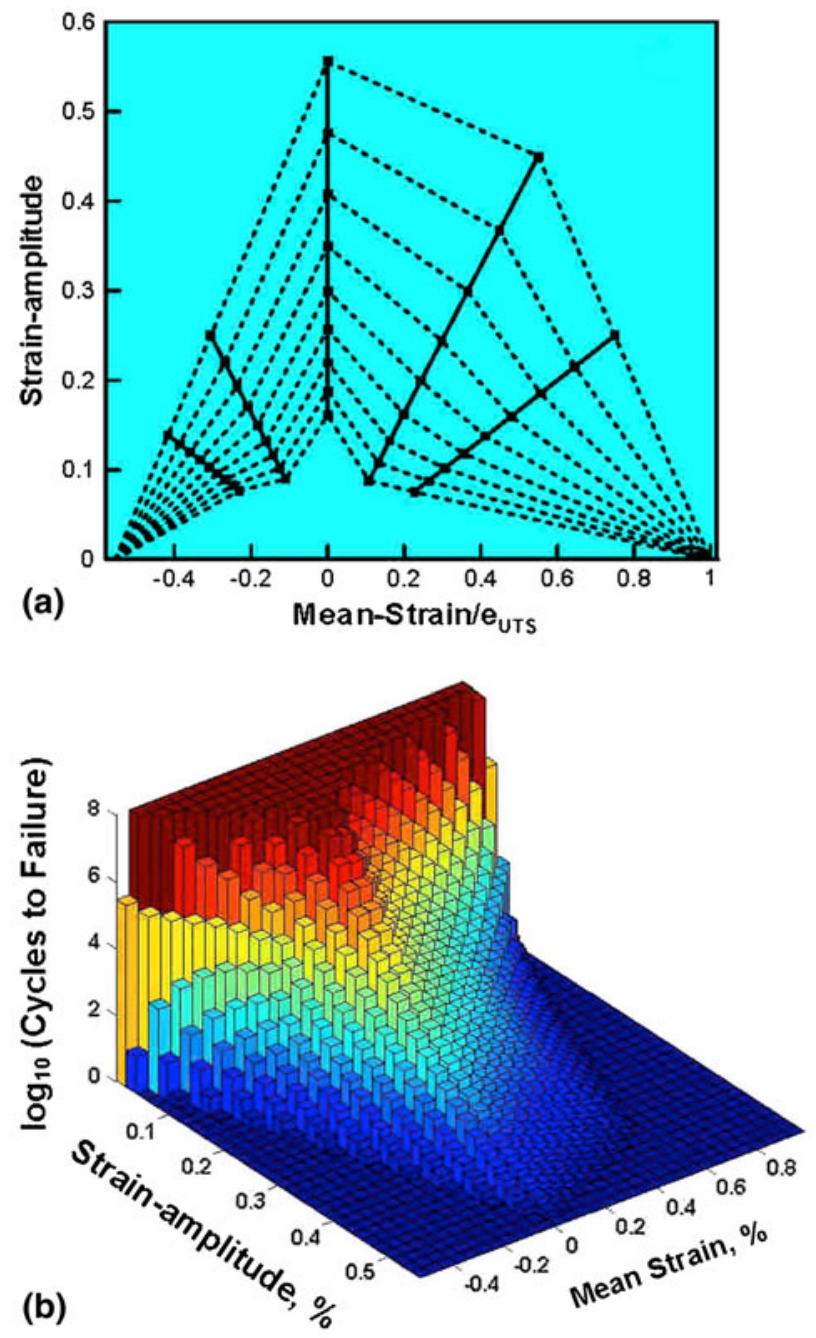

Fig. 12 An example of (a) Goodman diagram showing constant fatigue-life data (dashed lines) and constant R-ratio data (the solid lines emanating from the origin); and (b) the corresponding threedimensional histogram showing the effect of strain amplitude and the mean strain on the material fatigue life

by the associated total fractional damage. According to this procedure, fatigue-induced failure occurs when the total fractional damage reaches the value of 1.0.

3.4.4 Stent Service Life Preliminary Prediction. The rainflow cycle-counting algorithm, linearized Goodman diagram, and the Miner's rule described above when combined with the Nitinol fatigue data, Fig. 10(a) and (b), and the finite element results pertaining to the maximum principal strain amplitude and the maximum principal strain mean value histories, can be used to determine the number of cardiac cycles to failure at each location within the stent.

To determine the fatigue life of the stent, one must identify the location within the stent which is associated with the smallest number of cardiac cycles to failure. This is typically done by first identifying the "hot spots" within the stent and then by recording a detailed maximum principal strain history at these locations within the stent. An example of a typical maximum principal strain history at a single location within the stent during a single cardiac cycle is displayed in Fig. 13. It is seen that the strain history is generally more complex than the imposed loading history justifying the use of the rainflow 


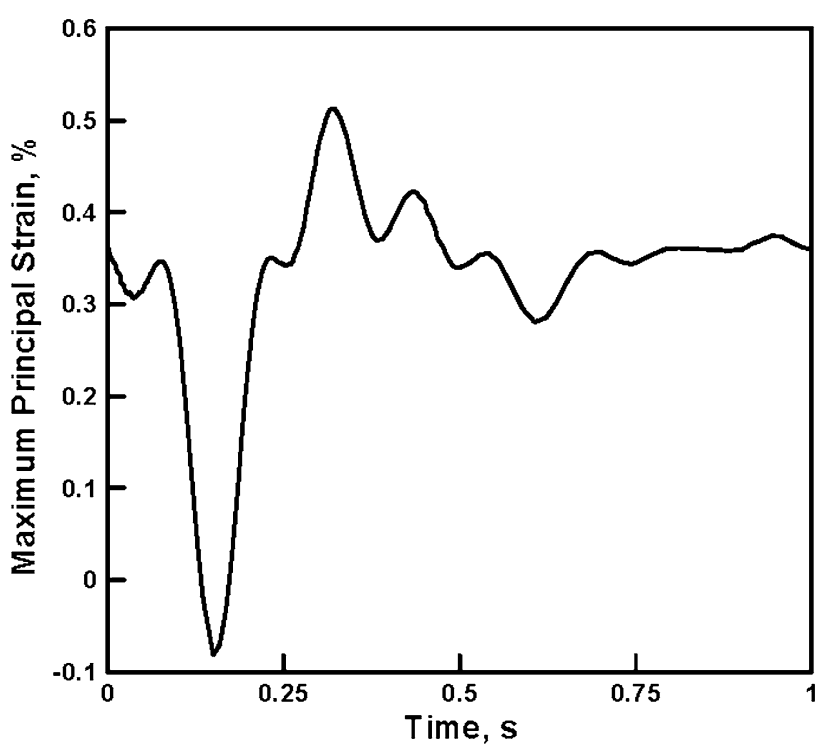

Fig. 13 Maximum principal strain history for a typical highly stressed location within the stent during a single cardiac cycle

cycle-counting algorithm. Next, for each of the monitored locations, the rainflow cycle-counting algorithm, linearized Goodman diagram, and the Miner's rule are employed to determine the respective number of cardiac cycles to failure. Finally, the location associated with the smallest number of cycles to failure is used to define the stent fatigue life.

This procedure is being used in our ongoing investigation, and the results will be reported in a future communication. In addition, the procedure introduced in the present study can be coupled with the conventional design optimization tools to identify topological and geometrical modifications in the stent design, which could increase the stent fatigue life. This approach is being pursued in the aforementioned ongoing investigation, and the results will also be reported in a future communication.

\section{Summary and Conclusions}

Based on the results obtained in the present study, the following main summary remarks and conclusions can be drawn:

1. Advanced computational methods and tools are combined with the fatigue-life prediction methodologies and the available material fatigue data to lay down the basis for the prediction of fatigue-controlled service life of selfexpanding endovascular Nitinol stents.

2. By employing both the purely structural and the fluid-structure interaction computational methods, it was concluded that for reliable stent life prediction, explicit modeling of the circulating blood flow is not required, and its effect can be accounted for by prescribing the appropriate cardiac-cycle-based pressure boundary conditions to the artery and by quantifying artery/stent contact interactions.

3. It is argued that the proposed computational-aided engineering approach can complement the conventional make-and-test approaches to reduce the cost of redesign of the endovascular stents with improved fatigue life.

\section{References}

1. W.J. Buehler and R.C. Wiley, inventors, Nickel-Based Alloys, USA Patent No. 3,147,851, 1965

2. J.P. Espinos, A. Fernandez, and A.R. Gonzalez-Elipe, Oxidation and Diffusion Processes in Nickel-titanium Oxide Systems, Surf. Sci., 1993, 295, p 402-410

3. S. Kujala, A. Pajala, M. Kallioinen, A. Pramila, J. Tuukkanen, and J. Ryhanen, Biocompatibility and Strength Properties of Nitinol Shape Memory Alloy Suture in Rabbit Tendon, Biomaterials, 2004, 25, p 353-358

4. J. Ryhanen, Biocompatibility of Nitinol, SMST-2000: The International Conference on Shape Memory and Superelastic Technologies (Pacific Grove, CA, USA, 2001), 2003, p 251-259

5. L.H. Yahia, Shape Memory Implants, Springer, Berlin, 2000

6. T. Duerig, A. Pelton, and D. Stockel, An Overview of Nitinol Medical Applications, Mater. Sci. Eng. A, 1999, 273, p 149-160

7. T.W. Duerig, D.E. Tolomeo, and M. Wholey, An Overview of Superelastic Stent Design, Minim. Invasive Therapy Allied Technol., 2000, 9, p 235-246

8. T.W. Duerig, Applications of Shape Memory, Materials Science Forum, in Martensitic Transformations II, Vol. 56, B.C. Muddle, Ed., Trans. Tech. Publications, 1990, p 679-692

9. T.W. Duerig, Present and Future Applications of Shape Memory and Superelastic Materials, Proc. Mater. Res. Soc. Symp., 1995, 360, p 497-506

10. T.W. Duerig and K.N. Melton, Applications of Shape Memory in the USA, New Materials and Processes for the Future, N. Igata and I. Kimpara, Ed., Society for the Advancement of Material and Process Engineering, Cleveland, OH, 1989, p 195-200

11. A.R. Pelton, D. Stoeckel, and T.W. Duerig, Medical Uses of Nitinol, Mater. Sci. Forum, 2000, 327-328, p 63-70

12. T.W. Duerig, K.N. Melton, C.M. Wayman, and D. Stoeckel, Engineering Aspects of Shape Memory Alloys, Butterworth-Heinemann Ltd., London, 1990

13. G.M. Michal and R. Sinclair, The Structure of TiNi Martensite, Acta Crystallogr. B, 1981, B37, p 1803-1806

14. G. Tan and Y. Liu, Comparative Study of Deformation-Induced Martensite Stabilization Via Martensite Reorientation and StressInduced Martensitic Transformation in NiTi, Intermetallics, 2004, 12, p 373-381

15. L. Tan and W.C. Crone, In situ TEM Observation of Two-Step Martensitic Transformation in Aged NiTi Shape Memory Alloy, Scr. Mater, 2004, 50, p 819-823

16. J. Uchil, K.K. Mahesh, and K.K. Ganesh, Calorimetric Study of the Effect of Linear Strain on the Shape Memory Properties of Nitinol, Physica B Condens. Matter, 2001, 305, p 1-9

17. T.W. Duerig, Some Unsolved Aspects of Nitinol, Mater. Sci. Eng. A, 2006, 438-440, p 69-74

18. T. W. Duerig and A. R. Pelton, "An Introduction to Nitinol Biomedical Devices," Workshop: Shape Memory and Superelastic Technologies Conference (SMST), Pacific Grove, CA, 2003

19. J.M. McNaney, V. Imbeni, Y. Jung, P. Papadopoulos, and R.O. Ritchie, An Experimental Study of the Superelastic Effect in a Shape-Memory Nitinol Alloy Under Biaxial Loading, Mech. Mater., 2003, 35, p 969986

20. M. Grujicic, Design of Precipitated Austenite for Dispersed-Phase Transformation Toughening in High Strength Co-Ni Steels, Mater. Sci. Eng. A, 1990, 128, p 201-207

21. R.H. Dauskardt, T.W. Duerig, and R. O. Ritchie, Effects of In Situ Phase Transformation on Fatigue-Crack Propagation in TitaniumNickel Shape-Memory Alloys, Proceedings of the MRS International Meeting on Advanced Materials, 9, Shape Memory Materials, Tokyo, Japan, 31 May-3 June, 1988-1989, p 243-249

22. R.L. Holtz, K. Sadananda, and M.A. Imam, Fatigue Thresholds of Ni-Ti Alloy Near the Shape Memory Transition Temperature, Int. J. Fatigue, 1999, 21, p S137-S145

23. A. Mehta, V. Imbeni, R.O. Ritchie, and T.W. Duerig, On the Electronic and Mechanical Instabilities in Ni50.9Ti49.1, Mater. Sci. Eng. A, 2004, 378, p 130-137

24. M. Simon, inventor, Blood Clot Filter, USA Patent No. 4,425,908, 1984

25. M.E. Ring, How a Dentist's Name Became a Synonym for a LifeSaving Device: The Story of Dr. Charles Stent, J. Hist. Dent., 2001, 49, p $77-80$ 
26. B.D. Ratner, A.S. Hoffman, F.J. Schoen, and J.E. Lemons, Ed. Biomaterials Science: An Introduction to Materials in Medicine, Academic Press, San Diego, CA, 1996

27. N. Rebelo, X. Gong, A. Hall, A.R. Pelton, and T.W. Duerig, Finite Element Analysis on the Cyclic Properties of Superelastic Nitinol, Proceedings of the International Conference on Shape Memory and Superelastic Technologies, Baden, Germany, 2004

28. N. Rebelo, R. Fu, and M. Lawrenchuk, Study of a Nitinol Stent Deployed into Anatomically Accurate Artery Geometry and Subjected to Realistic Service Loading, J. Mater. Eng. Perform., 2009, 18, p 655663

29. N. Rebelo, A. Zipse, M. Schlun, and G. Dreher, A Material Model for the Cyclic Behavior of Nitinol, J. Mater. Eng. Perform., 2011, 20, p 605-612

30. N. Rebelo, R. Radford, A. Zimpse, M. Schlun, and G. Dreher, On Modeling Assumptions in Finite Element Analysis of Stents, J. Med. Devices, 2011, 5, p 1-7

31. A.L. McKelvey and R.O. Ritchie, Fatigue-crack Propagation in Nitinol, A Shape-Memory and Superelastic Endovascular Stent Material, J. Biomed. Mater. Res., 1999, 47(3), p 301-308

32. A.R. Pelton, V. Schroeder, M.R. Mitchell, X. Gong, M. Barney, and S.W. Robertson, Fatigue and Durability of Nitinol Stents, J. Mech. Behav. Biomed. Mater., 2008, I, p 153-164

33. S.M. Harvey, Nitinol Stent Fatigue in a Peripheral Human Activity Subjected to Pulsatile and Articulation Loading, J. Mater. Eng. Perform., 2011, 20, p 697-705

34. ABAQUS/Standard, User Documentation, Dassault Systems, 2011

35. ABAQUS/Explicit, User Documentation, Dassault Systems, 2011
36. ABAQUS Version 6.10EF, User Documentation, Dassault Systems, 2011

37. F. Auricchio and R. Taylor, Shape-Memory Alloys: Modeling and Numerical Simulations of the Finite-Strain Superelastic Behavior, Comput. Methods Appl. Mech. Eng., 1996, 143, p 175-194

38. F. Auricchio and R. Taylor, Shape-Memory Alloys: Macromodeling and Numerical Simulations of the Superelastic Behavior, Comput. Methods Appl. Mech. Eng., 1997, 146, p 281-312

39. E.M. Tittelbaugh, R. Fu, and S. Sett, Coupling FEA to CFD to Investigate the Effects of Pulsatile Blood Flow on the Dilatation of Artery Walls, ABAQUS Users Conference, 2007

40. D.A. Steinman, B. Vinh, C.R. Ethier, M. Ojha, R.S.C. Cobbold, and K.W. Johnston, A Numerical Simulation of Flow in a Two-Dimensional End-to-Side Anastomosis Model, J. Biomed. Eng., 1993, 115, p 112-118

41. M. Matsuiski and T. Endo, Fatigue of Metals Subjected to Varying Stress, Japan Society of Mechanical Engineering, Tokyo, 1969

42. J. Goodman, Mechanics Applied to Engineering, Longman, Green, \& Co., London, 1899

43. M.A. Miner, Cumulative Damage in Fatigue, J. Appl. Mech., 1945, 12, p A159-A164

44. M. Grujicic, G. Arakere, E. Subramanian, V. Sellappan, A. Vallejo, and M. Ozen, Structural-Response Analysis, Fatigue-life Prediction and Material Selection for $1 \mathrm{MW}$ Horizontal-Axis Wind-Turbine Blades, J. Mater. Eng. Perform., 2010, 19(6), p 780-801

45. M. Grujicic, G. Arakere, B. Pandurangan, V. Sellappan, A. Vallejo, and M. Ozen, Multidisciplinary Optimization for Fiber-Glass Reinforced Epoxy-Matrix Composite $5 \mathrm{MW}$ Horizontal-Axis Wind-Turbine Blades, J. Mater. Eng. Perform., 2010, 19(8), p 1116-1127 